\title{
The role of the medical registrar
}

\author{
Paul Grant and Andrew Goddard
}

\begin{abstract}
The medical registrar in the acute on-call and outof-hours setting is usually considered to be one of the busiest and most challenging jobs in the entire hospital. This is perhaps a reflection of the changes in the structure and organisation of acute medicine precipitated by the European Working Time Directive and Modernising Medical Careers. As well as the general feeling that medicine is being increasingly viewed as a default referral option by other specialties who are themselves becoming ever more sub-specialised. This article explores what the pragmatic role of the medical registrar broadly should be. The Medical Workforce Unit at the Royal College of Physicians is launching an initiative, part funded by the Department of Health, to answer this difficult question.
\end{abstract}

KEY WORDS: acute medicine, European Working Time Directive (EWTD), general internal medicine, hospital at night, medical registrar, out of hours

How does one define the role of the medical registrar, that dynamic action-orientated problem solver, who is charged with leading the acute medical on-call, being the referring doctor for the entire hospital, a general practice helpline, counsellor for distressed relatives and gatekeeper of the medical assessment unit? Some registrars view the on-call day for medicine as a highlight of the working week, an opportunity to see more, do more, turn around acutely sick patients rapidly and see their efforts have a positive effect. They see the hectic and demanding workload as making the experience both more challenging and rewarding.

However, times are changing and this view is anything but widely held. The medical registrar in the acute on-call and outof-hours setting is usually considered to be one of the most difficult jobs in the whole hospital. Medicine is increasingly viewed as a dumping ground by other specialties, eg by surgeons - acute pancreatitis - 'no operation needed, refer to medics'; by accident and emergency - alcohol intoxication - 'observation only, refer to medics'; by psychiatrists - acute psychosis - 'need to rule out an organic cause first, refer to medics' and so on. ${ }^{1}$

Much appears to have changed regarding medical training and the development of new working practices in a relatively short space of time - consequent to the shadow of those familiar acronyms EWTD (European Working Time Directive) and MMC (Modernising Medical Careers). Firstly what is meant by 'new working practices'? What are the essential differences

Paul Grant, specialist registrar, general internal medicine and diabetes and endocrinology, King's College Hospital, London; Andrew Goddard, director, Medical Workforce Unit, Royal College of Physicians, London between new and old style hospital doctoring? Many would argue that a move to a shift-based or partial shift system has provoked the dissolution of the traditional firm structure, there is a reduction in the total number of hours worked and this leads to disjointed working - that is the dissonance between on-call working and 'normal' work which leads to loss of continuity of care for patients, more reliance on poor quality, rushed handovers and reduced ability for trainees to follow up their activities to aid learning about outcomes. ${ }^{2}$ The acute medical on-call and out-of-hours working represent prime opportunities for doctors in training to enhance their skills and knowledge at the sharp end and learn how to become autonomous, self-motivated, professionals - leading the medical take.

Working lives may be better and more balanced and certainly one could suggest that patient safety is enhanced by not having over-tired junior doctors potentially making clinical errors (and relating to this it is interesting to know the impact on the frequency and severity of medical errors before and after MMC and EWTD), but the gross total amount of good training opportunities has undoubtedly reduced. One has to consider the longer term implications of this change. McIntyre et al demonstrated that overall patient care did not significantly worsen before and after EWTD compliant changes, although rates of sick leave among junior doctors did. ${ }^{3}$ The theory being that the break up of the medical firm, a supportive, integrated team, had led to a disinclination for medical professionals to go that extra mile and support their colleagues. Shift working is bitty, disaggregated and oppositional to traditional team working. The loss of feedback from a shared experience can also lead to reduced job satisfaction. ${ }^{4}$

It is difficult then to understand and agree on the definition of the clinical and non-clinical activities of the medical registrar working in UK hospitals, at both a teaching and district general level. How much should the registrar contribute to the hospital at night team? What are the areas of good and bad practice that can be identified to improve both the quality of patient care and the standard of medical training? To this end, the Medical Workforce Unit at the Royal College of Physicians (RCP) is planning to explore what the pragmatic role of the medical registrar should broadly be. An initiative, part-funded by a grant from the Department of Health, has now been launched which will run over the next year and investigate the many varied tasks of the medical registrar in the out-of-hours setting. Specific areas to be studied include:

- time spent on various clinical activities, eg new patient clerking, requesting tests, reviewing patients, supervision of juniors, general practitioner phone calls, by means of diaries, questionnaires and interviews 
- interactions of the medical registrar with members of other clinical teams, with respect to time, clinical utility and educational value

- the nature and impact of teaching: time spent, modalities used, subjective and objective measures of effectiveness

- the role of the medical registrar in handover of patients between working shifts (building on work that has already been done by the RCP)

- the perception of the role of the medical registrar by other doctors, healthcare professionals as well as patients (via the RCP's patient carer network).

Two current medical registrars have been employed as clinical fellows by the RCP Medical Workforce Unit to work on this exciting and relevant project. They will collect information through electronic questionnaires, interviews and visits to hospital trusts throughout England in order to cover as many of the strategic health authority regions as possible. The ultimate goal is to get a picture of the disparate work that takes place under the large umbrella of the medical registrar. What are the attitudes of medical registrars and those that work alongside them to the work that they undertake and its appropriateness? Are there areas of best practice that we can share and learn from? Are there lines to be drawn around what is and is not acceptable for the beleaguered medical registrar in the middle of the night and is damage being done to the specialty of general medicine because of negative perceptions?
This important survey will assess the pivotal role of the medical registrar and its findings, in particular the assessment of different models of working, handover, interaction with other healthcare professionals, as well as the impact on training, are eagerly anticipated. This should make things better for both patients and the specialty trainees on the shop floor.

\section{Acknowledgments}

Many thanks to Dr Ian Scobie, Derek Lington and Stephen Morris.

\section{References}

1 Houghton M. Acute medicine - an alternative take. Clin Med 2011;11:26-7.

2 NHS Medical Education England. Review of the impact of the European Working Time Directive (EWTD) on the quality of postgraduate training. Consultation exercise 2009-2010. London: NHS Medical Education England, 2010. www.aukuh.org/index.php/component/ docmandoc_download/30-ewtd-review

3 McIntyre HF, Winfield S, Te HS, Crook D. Implementation of the EWTD in an NHS trust: impact on patient care and junior doctor welfare. Clin Med 2010;10:2:134-7.

4 Grant P. Physician job satisfaction in the United Kingdom vs. New Zealand. NZMJ 2004;117:U1123.

Address for correspondence: Dr P Grant, King's College Hospital, Denmark Hill, London SE5 9RS.

Email: drpaul.grant@doctors.org.uk 